\title{
EDITORIAL
}

\section{Emerging epidemic of noncommunicable diseases}

Noncommunicable diseases (NCDs) are the leading cause of death globally, and one of the major health challenges of the 21 st century. In September 2011, at the United Nations General Assembly in New York, a political declaration was made to strengthen global and national responses to prevent and control NCDs. ${ }^{1} \mathrm{~A}$ change in the disease profile from communicable diseases to NCDs in low and middle income countries resulted in the inclusion of NCDs in the 2030 agenda for sustainable development. The definition of NCDs has also been broadened with the move away from the " $4 \mathrm{x} 4$ approach" (four major NCDs and four modifiable risk factors) to the " $5 \times 5$ approach", which includes mental health and air pollution. ${ }^{2}$

In 2016, NCDs are estimated to account for $71 \%$ of the 57 million global deaths. The major NCDs responsible for these deaths included cardiovascular diseases (17.9 million deaths, accounting for $44 \%$ of all NCD deaths and $31 \%$ of all global deaths); cancers (9 million deaths, $22 \%$ of all NCD deaths and $16 \%$ of all global deaths); chronic respiratory diseases $(3.8$ million deaths, $9 \%$ of all NCD deaths and $7 \%$ of all global deaths); and diabetes (1.6 million deaths, $4 \%$ of all NCD deaths and $3 \%$ of all global deaths). Air pollution was the second largest risk factor causing NCDs globally, just after tobacco smoking but in southeast Asia it is by far the largest cause of NCDs.

A clear relationship is evident between premature NCD mortality and country income levels. More than three quarters $(78 \%)$ of NCD mortality was in low and middle income countries; $47 \%$ of these deaths were premature, affecting people aged 30-70 years. These levels are growing steadily. NCD mortality also varied by WHO region, with a greater probability observed in the African $(22 \%)$, Eastern Mediterranean (24\%) and South East Asian (23\%) regions, compared with the Region of the Americas (15\%), and the European (17\%) and Western Pacific $(16 \%)$ regions. In all WHO regions, the probability of dying from an NCD was greater for males than for females. ${ }^{3}$

According to 2016 data from Bangladesh total population was $163,000,000$ and total death was $8,56,000$ and $21 \%$ of total death was premature death. 4 NCDs accounted for $67 \%$ of all deaths (30\%-CVD, $12 \%$ Cancer, 10\%-COPD, 3\%-Diabetes, 12\%-other NCDs). Other causes are, 26\%- communicable, perinatal \& Nutritional diseases and $7 \%$ injuries. Among these, 66,400 lives can be saved by 2025 by implementing all of the WHO 'best buys'.

NCD's are chronic conditions characterized by complex etiology, multiple risk factors, long latency period, noncontagious, prolonged course of illness and functional impairment. Most African and Asian countries are undergoing an epidemilogical transition, which is a shift from a pattern of predominantly infectious diseases to that of chronic non-communicable diseases particularly in low and middle income countries, which already bear an appreciable burden of communicable diseases (a double burden of disease). This is as a result of urbanization, industrialization, increased life expectancy and the adoption of western lifestyle characterized by reduced physical activity and dietary changes from foods rich in fruits and vegetables to refined, energy dense and fatty foods. ${ }^{5}$

Current evidence shows that four major groups of diseases namely cardiovascular diseases, cancers, respiratory diseases and diabetes mellitus account for $82 \%$ of all NCD deaths. These diseases share four common behavioural risk factors (tobacco use, excess alcohol consumption, unhealthy diet and physical inactivity) and four metabolic risk factors (elevated blood pressure, overweight and obesity, hyperglycemia and hyperlipidaemia). The World Health Organization has recommended the surveillance of NCDs and their risk factors to inform the implementation of appropriate public health strategies. The, WHO Stepwise approach is a standardized methodology for the surveillance of non communicable diseases (STEPS).

National burden of NCD's is nearly similar to global burden of diseases. An estimated 17.3 million people died from CVDs in 2008, (30\% of all global deaths) 7.3 million were due to coronary heart disease $\& 6.2$ million were due to stroke. Over $80 \%$ CVD deaths occur in low and middle income countries. By 2030, almost 25 million people will die from CVDs. Ischaemic heart disease (IHD) has become the third killer disease in Bangladesh. Prevalence of IHD and stroke in Bangladesh population is $2.2 \%$ and $1.7 \%$ respectively. A rural clinic based data showed the prevalence of hypertension to be $12 \%{ }^{1}$

Globally, 7.6 million people died from cancer in 2008 . Seventy percent of all cancer deaths occur in low and middle income countries. Deaths from cancer are estimated to reach 13.1 million by 2030. A hospital based registry in the National Institute of Cancer Research and Hospital indicates that 11,903 new cancer patients attended the outpatient departments in 2005-6. The highest frequency is observed for lung cancer $(24.1 \%)$ in men followed by breast cancer $(23.7 \%)$ and cervical cancer $(22.8 \%)$ in women. High incidence of cervical cancer in Bangladesh is related to early marriage, multiparity, and low socioeconomic conditions. Sixty five million people worldwide have moderate to severe COPD. More than 3 million people died of COPD in 2005 ( $3 \%$ of all deaths globally). Almost $90 \%$ of COPD deaths occur in low and middle income countries. The National Institute of Diseases of Chest and Hospital, admits about 4500 patients annually in the department of respiratory medicine, of them 19\% suffer from COPD. Smoking and indoor air pollution are thought to be the two most important causes of COPD in Bangladesh. ${ }^{4}$

In 2004, an estimated 3.4 million people died from consequences of uncontrolled Diabetes Mellitus. More than $80 \%$ of diabetes deaths occur in low and middle income countries. WHO projects that diabetes death will increase by two thirds between 2008 and 2030. From the hospital record of BIRDEM, it was found that number of diabetic patients has been increasing exponentially since 
1960. In rural adults, the prevalence is about $5 \%$, and in urban area the prevalence is just double $(10 \%)$. This could reflect the effect of unplanned urbanization that lacks in environment for physical activity, consumption of junk food and exposure to stressful life in cities.

The nation wide community based Bangladesh Health and Injury Survey (BMS) reported an estimated 70,000 annual deaths due to injury that includes 30,000 children of under 18 years. Road traffic injuries is gradually worsening in Bangladesh due to unplanned urbanization and chaotic traffic system. Drowning is also common injury in Bangladesh and have become a major concern particularly in children.

Much of the global change in NCDs has been driven by population growth and ageing in the past decade, increasing the population vulnerable to NCD determinants. Several risk factors are implicated with NCDs.

A nonmodiflable risk factors cannot be reduced or controlled by intervention; for example: Age, Gender, Race, and Family history (genetics). A modifiable risk factors are behavioral factor that can be reduced or controlled by intervention, thereby reducing the probability of disease. WHO has prioritized the following four: Physical inactivity, Tobacco use, Alcohol use, and unhealthy diets (increased fat and sodium, with low fruit and vegetable intake). ${ }^{5}$

Metabolic factors (modifiable risk factors) can lead to metabolic changes in the body. WHO has prioritized the following four metabolic risk factors: Raised blood pressure, Raised total cholesterol, Elevated glucose, Overweight and obesity.

Raised BP is the leading risk factor for stroke, coronary heart disease. In some age groups, the risk of CVD doubles for each increment of $20 / 10 \mathrm{mmHg}$ of blood pressure. High sodium intake, through hypertension, is a major cause of cardiovascular disease, death and disability. About $10 \%$ of cardiovascular disease is caused by excess sodium intake. A population salt intake of less than 5 grams or approximately 2,000 milligrams of sodium, per person per day is recommended to reach the national targets.

Globally, $1 / 3 \mathrm{rd}$ of ischaemic heart disease is attributable to high cholesterol. A $10 \%$ reduction in serum cholesterol in men aged 40 has been reported to result in a $50 \%$ reduction in heart disease within 5 years. In 2004 , it was estimated that elevated glucose resulted in 3.4 million deaths $(5.8 \%$ of all deaths $)$. Globally, approximately $9 \%$ of adults aged 25 and over had elevated blood glucose in 2008.

Worldwide, obesity has more than doubled since 1980 . In 2008 , more than 1.4 billion adults were overweight. $65 \%$ of the world's population live in countries where the mortality associated with overweight and obesity is higher than the mortality associated with underweight.

The UN Agenda for Sustainable Development goal is the driving force for high level political commitment and accountability to achieve the targets, including reducing premature mortality from NCDs by $30 \%$ by 2030 . The paradigm of NCD prevention is considered as a multidisciplinary one. It necessitates a diverse range of actions involving policy development, legislation, regulation, public and professional education, guideline development and media interventions. Monitoring, evaluation and research are required to be woven into this framework. ${ }^{4}$ Each Government has the central role, in cooperation with other stakeholders, to create an environment that empowers and encourages behavior changes by individuals, families and communities. Substantial external investments will also be required to strengthen the ministries of health in low income countries to ensure the development and full implementation of national strategies. ${ }^{2}$

The WHO Stepwise approach is a standardized methodology for the surveillance of non communicable diseases, Step 1- involves the use of questionnaire to collect information on socio demographic characteristics, behavioral risk factors and metabolic risk factors; Step 2- Anthropometric measurements - Height, Weight, Body mass Index (BMI), Waist circumference; Step 3Biological measures -Blood pressure measurements \& Fasting blood sugar screening. WHO identified a package of 16 "best buy" interventions that are cost effective, affordable and feasible in all settings under the banner of - Reduce Tobacco use; Reduce alcohol use; Reduce unhealthy diet; Reduce physical inactivity; Manage CVD \& Diabetes and Manage Cancer.

Bangladesh has made considerable progress in the past decades in improving the health of the population. As a result early demographic transition have become apparent in the age structure of the population.4 Now the Government has given special attention to tackle NCDs, at the primary care level and accepted WHO-PEN protocol for early detection and management of chronic diseases and its complications. The action plan has also been adopted based on the four strategic priority areas outlined in the WHO plan for the prevention and control of noncommunicable diseases in South-East Asia. Public awareness through different media could be immensely helpful in scaling up the intervention process.

CH Rasul, Professor of Paediatrics, KCMC\&H (Email: chrasul@yahoo.com)

\section{References:}

1. World Health Organization. Noncommunicable disease Fact sheet. 2018. www.who.int/newsroom/ fact sheets/detail/ noncommunicable diseases.

2. Collins T. Time to align: development cooperation for the prevention and control of non-communicable diseases. BMJ 2019; 366:14499: 1-5

3. Bigna. JJ, Noubia JJ. The rising burden of non communicable diseases in sub-Saharan Africa. The Lancet 2019; 7: e1296

4. DGHS \& MoHFW. Multisectoral action plan for prevention and control of noncommunicable diseases 2018-2025. NCDC program, Dhaka 2018

5. United Nations System Standing Committee on Nutrition. Non communicable diseases, diets and nutrition 2018. www.unscn.org/uploads/web/news/document/NC Ds-brief EN-WEB.pdf 